\title{
Brote de pseudofungemia por Fusarium spp. en una unidad oncológica infantil
}

\author{
Outbreak of pseudofungemia by Fusarium spp. \\ in a pediatric oncology unit \\ Marisol Tamayo-Vélez MSc ${ }^{1}$, Santiago L. Atehortúa-Muñoz MSc², \\ Lina M. Echeverri-Toro MD²
}

Introducción: dentro del comportamiento microbiológico de una unidad oncológica pediátrica en Medellín, Colombia, se detectó una presentación inusual de casos de fungemia por Fusarium spp. en un corto periodo. Objetivo: describir un brote hospitalario causado por Fusarium spp. en pacientes pediátricos hospitalizados en una unidad oncológica en Medellín, Colombia. Materiales y métodos: se realizó un estudio descriptivo sobre un brote hospitalario por Fusarium spp. aislado en sangre de cinco pacientes pediátricos hospitalizados en una unidad de oncología en Medellín, Colombia, entre diciembre de 2012 y febrero de 2013. Resultados: cuatro de los cinco pacientes con hemocultivos positivos para Fusarium spp. tenían acceso venoso central y en tres de ellos el único hemocultivo positivo fue tomado a través de una de las vías del catéter. Dado lo anterior, sumado a que no había correlación con los hallazgos clínicos, tres pacientes se consideraron casos de pseudofungemias y no se les administró tratamiento antifúngico. Dentro de las hipótesis planteadas sobre la causa del brote se confirmó la contaminación del ambiente con el hongo debido a un sistema de ventilación ineficiente.

\footnotetext{
${ }^{1}$ Médica y Cirujana, Especialista en Auditoría en Salud, MSc en Epidemiología. Epidemióloga, Oficina de Garantía de la Calidad y Vigilancia Epidemiológica, Hospital Universitario de San Vicente Fundación. Medellín, Colombia. Correspondencia: Calle 64 \#51 D-154. Teléfono: 5744441333 ext. 3110-3115. Correo electrónico: marisoltamayo.velez@gmail.com,mtvx@sanvicentefundacion.com

2 Médicos y cirujanos, MSc en Ciencias Médicas (énfasis Microbiología). Médicos microbiólogos, Microbiología Clínica, Hospital Universitario de San Vicente Fundación. Medellín, Colombia.

Conflicto de intereses: los autores declaran que no tienen conflicto de intereses Medicina \& Laboratorio 2016; 22: 239-252

Módulo 19 (Investigación), número 45. Editora Médica Colombiana S.A. $2016^{\circ}$

Recibido el 05 de junio de 2016; aceptado el 27 de junio de 2016
} 
Conclusiones: en la mayoría de los casos del brote Fusarium spp. se consideró contaminante de los dispositivos intravasculares y se asoció a dificultades en el control ambiental de las habitaciones.

Palabras clave: seguridad del paciente, exposición a riesgos ambientales, malignidades hematológicas, infección hospitalaria, Fusarium, pseudofungemia.

Introduction: An unusual presentation of cases of fungemia by Fusarium spp. was detected in pediatric oncology unit of Medellin, Colombia, in a short period. Objective: To describe a hospital outbreak caused by Fusarium spp. in pediatric hospitalized patients in an oncology unit of Medellin, Colombia. Materials and methods: A descriptive study of a hospital outbreak by Fusarium spp. isolated in blood cultures of five pediatric patients hospitalized in an oncology unit of Medellin, Colombia, between December 2012 and February 2013. Results: Four of the five patients with positive blood cultures for Fusarium spp. had central venous access, and in three of them, the unique positive blood culture was taken through one of the catheter tracks. Because of this and considering that there was no correlation with the clinical findings, three cases were determined as pseudofungemia and the antifungal treatment was not administered. Among the hypotheses raised about the outbreak cause, contamination of the environment with the fungus was confirmed due to an inefficient ventilation system. Conclusions: In most outbreak cases, Fusarium spp. was considered a contaminant of intravascular devices and it was associated with difficulties in rooms' environmental controls.

Key words: Patient Safety, environmental exposure, hematologic malignancies, cross infection, Fusarium, pseudofungemia.

Tamayo-Vélez M, Atehortúa-Muñoz SL, Echeverri-Toro LM. Brote de pseudofungemia por Fusarium spp. en una unidad oncológica infantil. Medicina \& Laboratorio 2016; 22: 239-252.

E n pacientes con neoplasias hematológicas las infecciones producidas por Candida spp. y Aspergillus spp. continúan siendo las más prevalentes, aunque cada vez son más frecuentes las infecciones causadas por hongos más inusuales como Fusarium spp., Trichosporon spp. y Scedosporium spp., entre otros [1].

Las especies del género Fusarium son comúnmente encontradas en el suelo y restos de material orgánico y con frecuencia causan enfermedad en las plantas. La enfermedad en humanos es poco frecuente y ocurre generalmente después de la inoculación traumática en el hospedero sano o por inhalación o traumatismos menores en pacientes inmunocomprometidos [2].

En el examen directo las hifas de Fusarium spp. se asemejan a las de Aspergillus spp. en tamaño, patrón de ramificación, septos y predilección por la invasión vascular. A partir de los cultivos el rasgo microscópico característico de Fusarium spp. 
es la producción de macroconidias septadas en forma de hoz o de plátano, que ayudan a su identificación [3].

Las formas clínicas de la fusariosis dependen del estado inmune del hospedero y la puerta de entrada de la infección. Fusarium spp. en el hospedero inmunocompetente puede causar lesiones localizadas en la córnea, la piel y las uñas, e infección de heridas. Entretanto, en los hospederos inmunocomprometidos produce infecciones diseminadas, con frecuente compromiso cutáneo en forma de lesiones nodulares tipo ectima gangrenoso. Este hongo es altamente angioinvasivo y se identifica fácilmente en la sangre por su capacidad para liberar conidios. También se han descrito infecciones asociadas a catéter, neumonía, sinusitis, infección rinocerebral, endoftalmitis, miositis, entre otras [1].

En contraste con la aspergilosis, en la que los resultados de los hemocultivos son casi siempre negativos, la fusariosis se acompaña de cultivos de sangre positivos en más del $50 \%$ de los pacientes que tienen la infección. De esta manera, el aislamiento del hongo a partir de muestras de sangre y la biopsia de piel de las lesiones sospechosas son las dos formas más comunes y eficaces para diagnosticar la fusariosis $[2,3]$. El objetivo de este artículo es informar sobre un brote hospitalario de pseudofungemia causado por aislamientos de Fusarium spp. en pacientes pediátricos hospitalizados en una unidad oncológica de la ciudad de Medellín, Colombia.

\section{Materiales y métodos}

\section{Diseño del estudio}

Se realizó un estudio descriptivo de un brote hospitalario por Fusarium spp. aislado en sangre de pacientes pediátricos hospitalizados en la unidad de Oncología Infantil del Hospital Universitario de San Vicente Fundación, Medellín, Colombia, entre diciembre de 2012 y febrero de 2013.

\section{Pacientes}

La toma de los hemocultivos se realizó según los protocolos establecidos por la institución. Las muestras de todos los pacientes se procesaron según los protocolos del Laboratorio de Microbiología, siguiendo la metodología convencional en el equipo automatizado BacT/Alert ${ }^{\circledR}$ (BioMérieux, Marcy-I'Étoile, Francia).

\section{Investigación sobre la fuente del brote}

Las hipótesis planteadas sobre la fuente del hongo fueron:

1. Insumo contaminado que se utiliza para la toma de las muestras para hemocultivos, tales como clorhexidina o yodo, jeringas, agujas, entre otros. 
2. Las manos del personal de la salud encargado de la toma de las muestras o manipulación de dispositivos intravasculares.

3. El sistema de conducción del aire, que finalmente contamina el ambiente, y los elementos relacionados con la infraestructura de los cuartos de esta sala.

Para descartar las hipótesis se realizaron cultivos microbiológicos de algunos antisépticos, medicamentos e insumos como jeringas, guantes, punzones para solución salina, entre otros. Posteriormente, se descartó como fuente del hongo el personal asistencial a través de la revisión clínica del $100 \%$ del personal médico y de enfermería que labora en este servicio, descartando afecciones ungueales o del canal auditivo externo que fueran sugestivas de una infección micótica.

Para evaluar la tercera hipótesis, relacionada con la infraestructura de las habitaciones y el servicio en general, se realizó una revisión de la parte locativa de las habitaciones. Finalmente, con base en los hallazgos obtenidos se realizaron cultivos de la superficie de las rejillas de extracción del aire acondicionado y el aire ambiental en las habitaciones donde estuvieron los pacientes con aislamiento microbiológico en sangre de Fusarium spp.

\section{Cultivos de superficies, insumos, soluciones y aire ambiental}

Las muestras de superficies e insumos se tomaron con la técnica con hisopo estéril humedecido con tioglicolato, frotando la superficie con este e introduciéndolo en el medio líquido de enriquecimiento que se incuba durante 24 horas. Luego se realizó repique en medios sólidos para hongos (agar Sabouraud, BioMérieux), los cuales fueron incubados a $37^{\circ} \mathrm{C}$ durante dos semanas.

El estudio de las soluciones se hizo inoculando una muestra de $3 \mathrm{~mL}$ de la solución a estudiar en medio de cultivo líquido (tioglicolato) durante 24 horas y, posteriormente, se sembraron en los medios de cultivo sólidos para hongos (agar Sabouraud), los cuales se incubaron a $37^{\circ} \mathrm{C}$ durante dos semanas.

Las muestras de aire ambiental se tomaron con la metodología de impacto por aspiración, en la cual se toma muestra de grandes volúmenes de aire $\left(1.000 \mathrm{~m}^{3}\right)$ en 10 minutos con el equipo Air Ideal ${ }^{\circledR}$ (BioMérieux), directamente sobre el medio de cultivo sólido. Se utilizaron medios para hongos (agar Sabouraud) incubados a $37^{\circ} \mathrm{C}$ durante dos semanas. En cada habitación se tomaron tres muestras con el mismo filtro estéril (iniciando en el centro de la habitación, luego en la esclusa y por último en el baño) en medios de cultivo independientes. El resultado de los cultivos de aire fue reportado en UFC $/ \mathrm{m}^{3}$ (Unidades Formadoras de Colonias $/ \mathrm{m}^{3}$ ).

La identificación fenotípica se realizó en el laboratorio clínico de la institución y en los casos con alguna dificultad se remitió la cepa para identificación en un laboratorio especializado en Micología de la ciudad. 


\section{Resultados}

\section{Descripción de la Unidad de Oncología Pediátrica}

La Unidad Oncológica Pediátrica está conformada por doce habitaciones individuales y tres habitaciones para alojamiento bipersonal. De las doce habitaciones individuales, seis son consideradas como áreas de ambiente controlado, es decir, son salas con las estructuras e instalaciones específicas y los parámetros adecuados para controlar la biocontaminación. Estas habitaciones cuentan con sistema de ventilación especial con presión positiva independiente para cada cuarto, que consiste en sistemas de inyección de aire con tres filtros en serie, el último de estos un HEPA 99\% (alta eficiencia en la extracción de partículas) y la extracción del aire se realiza a través de extractores ubicados en el baño y en la habitación.

Las habitaciones con presión positiva están sometidas a un mantenimiento mensual que incluye: medición y ajuste de parámetros técnicos, el cambio del prefiltro, la inspección de posfiltros (cambiados cuando se requiere) y la constatación de la adecuada operación de los equipos que miden el diferencial de presión entre el cuarto y la esclusa. Las demás habitaciones individuales no cuentan con las condiciones previamente expuestas. Las habitaciones de ambiente controlado en ocasiones no son suficientes para albergar la cantidad de pacientes neutropénicos que las requieren; por lo tanto, algunos de estos pacientes son hospitalizados en las otras habitaciones individuales. De manera rutinaria no se toman cultivos ambientales en estas habitaciones.

\section{Comportamiento microbiológico en la Unidad de Oncología Pediátrica}

Entre diciembre de 2012 y febrero de 2013 se obtuvieron cinco aislamientos de Fusarium spp. a partir de sangre de pacientes pediátricos hospitalizados en la Unidad de Oncología Infantil del Hospital Universitario de San Vicente Fundación, Medellín, Colombia; situación que no representa el comportamiento habitual de aislamiento de este microorganismo en el servicio (véase figura 1).

\section{Características de los pacientes con aislamiento de Fusarium spp.}

Los hemocultivos con resultados positivos para Fusarium spp. fueron solicitados por el médico tratante siguiendo el protocolo establecido en la institución para pacientes oncológicos con neutropenia febril. Algunas de las características clínicas y microbiológicas de estos pacientes se presentan en las tablas $\mathbf{1}$ y $\mathbf{2}$. Ninguno de ellos falleció como consecuencia de la infección por Fusarium spp. durante la vigilancia del brote. 


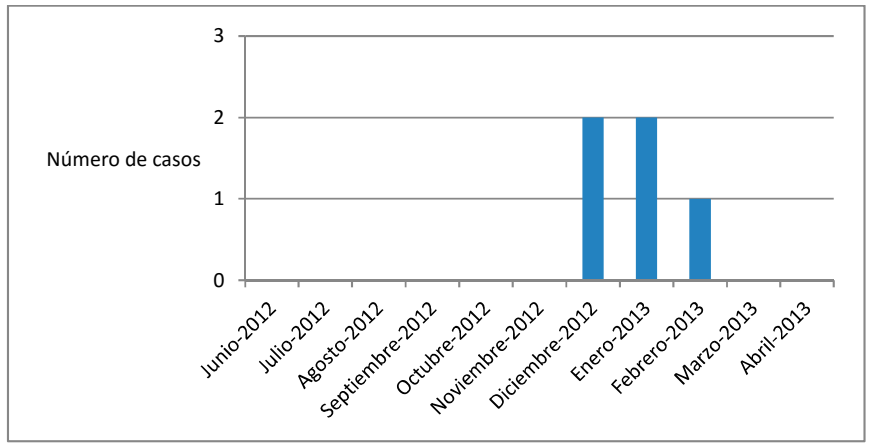

Figura 1. Número de casos de aislamiento microbiológico de Fusarium spp. en sangre de pacientes de la unidad de Oncología Infantil del Hospital Universitario de San Vicente Fundación, Medellín, Colombia, por mes de diagnóstico. Nota: en el mes de marzo de 2013 ocurrió el cierre del servicio.

\section{Tabla 1. Características de los pacientes con hemocultivos positivos para} Fusarium spp.

$\begin{array}{llllll}\text { Paciente }\left(\mathrm{N} .^{\circ}\right) & 1 & 2 & 3 & 4 & 5 \\ \text { Sexo } & \text { Masculino } & \text { Masculino } & \text { Femenino } & \text { Femenino } & \text { Masculino } \\ \text { Edad (años) } & 7 & 14 & 1 & 1 & 13 \\ \text { Tipo de malignidad } & \begin{array}{l}\text { Leucemia } \\ \text { mieloide } \\ \text { aguda }\end{array} & \begin{array}{l}\text { Linfoma de } \\ \text { Burkitt }\end{array} & \begin{array}{l}\text { Tumor ma- } \\ \text { ligno renal } \\ \text { rabdoide }\end{array} & \begin{array}{l}\text { Leucemia mie- } \\ \text { loide aguda }\end{array} & \begin{array}{l}\text { Leucemia } \\ \text { bifenotípica } \\ \text { refractaria a la } \\ \text { quimioterapia }\end{array}\end{array}$

Neutropenia* al

momento del aislamiento

de Fusarium spp.

Sí Sí

Sí Si

Sí Sí

Sí

Secreción en el sitio de inser-

Manifestaciones

Nódulos subcutáneos ción del catéter cutáneas de infección en cuero No por Fusarium spp. cabelludo No con cultivo del 19/01/13 positivo para Fusarium spp.

Manifestaciones por Fusarium spp.

Tratamiento con antifúngicos pulmonares de infección N
No Voriconazol por 15 días (inicio el 17/12/12)

No

Fluconazol 7 días (inicio el 16/12/12)

Biopsia de piel negativa para hongos

Comentarios Recibió voriconazol por fungemia
Recibió fluconazol por mucositis
No

Vorico-

nazol 21

días (inicio 09/01/13)

Recibió voriconazol por aspergilosis pulmonar
No

Voriconazol 10 días (inicio 20/01/13)

Recibió voriconazol por infec- Recibió flución del sitio de conazol por inserción del mucositis catéter

Fluconazol 8 días (inicio el 16/02/13)
Falleció por enfermedad de base

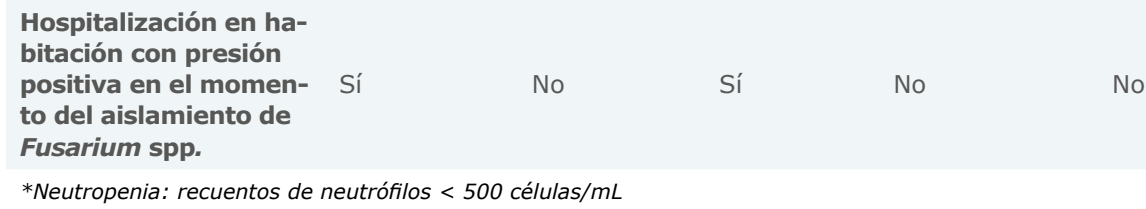




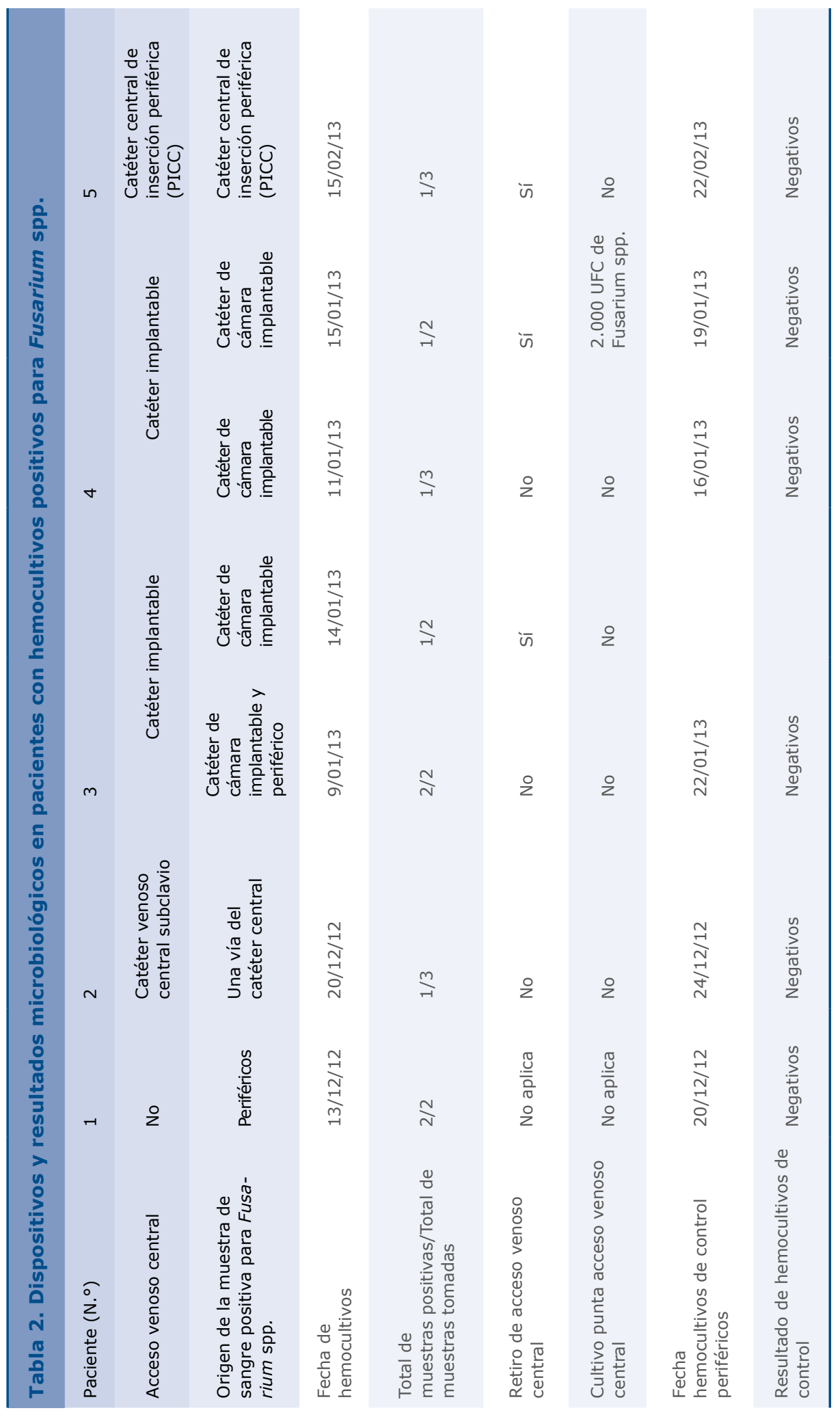




\section{Hallazgos de la investigación del brote}

Para determinar la fuente del hongo se realizaron un total de 27 cultivos microbiológicos de antisépticos, medicamentos e insumos utilizados en el Servicio de Oncología Pediátrica, todos ellos con resultados negativos. Además, ninguno de los miembros del personal médico y de enfermería de la Unidad presentó afecciones ungueales o del canal auditivo externo que fueran indicativas de una infección micótica. Descartadas estas hipótesis, se evaluó la tercera de ellas sobre la infraestructura de las habitaciones y el servicio en general, para la que se obtuvieron los siguientes resultados:

- En las habitaciones individuales sin presión positiva se encontró humedades en el cielo falso de los baños (material drywall) y una estructura del cielo falso no continua (no "junta perdida").

- En las habitaciones de ambiente controlado hubo algunos hallazgos de falta de higiene, principalmente en las rejillas de inyección y extracción del aire acondicionado, disrupciones en la unión entre la lámpara y el cielo falso y manejo inadecuado del sistema de ventilación de las habitaciones con presión positiva, como dejar abiertas las puertas de la habitación o de la esclusa.

- Para aislar el calor se encuentra encima del cielo falso un material en fibra de vidrio que en algunas habitaciones era incluso visible a través del espacio entre las lámparas y el cielo falso.

Los cultivos por hisopado de las rejillas de extracción del aire acondicionado y aire ambiental fueron realizados en las habitaciones donde estuvieron los pacientes con aislamiento de Fusarium spp. a partir de hemocultivos, dos con ambiente controlado y una sin presión positiva. En todas las habitaciones muestreadas se obtuvo un número variable de colonias (entre 1 y 10) de hongos como: Penicillium spp., Cladosporium spp., Fusarium spp., Aspergillus flavus, Aspergillus fumigatus y Aspergillus niger. Los resultados iniciales de los cultivos del aire en estas habitaciones se reportan en la tabla 3 que se presenta más adelante.

\section{Medidas de control del brote}

\section{Intervención inicial}

Teniendo en cuenta los resultados de los cultivos ambientales y de superficies se procedió a realizar como medida de intervención inicial: a) revisión del sistema de filtros de las habitaciones con cambio de los mismos, b) proceso de limpieza exhaustiva de las rejillas de inyección y extracción de aire, c) revisión de las puertas de las habitaciones dado que algunas no ajustaban adecuadamente, d) cierre hermético de los espacios entre las lámparas y el cielo falso, e) pintura y f) aseo general de las habitaciones. 
Posteriormente se realizó análisis microbiológico de la calidad del aire, el cual no mostró las condiciones óptimas para la hospitalización de los pacientes neutropénicos, lo que hizo necesario la evacuación del servicio para hacer la reestructuración necesaria.

\section{Intervención final}

La intervención final se enfocó en retirar el material usado para realizar el control del calor (fibra de vidrio), pues se encontró que retenía mucho polvo, y en cambiar el cielo falso de todo el servicio por uno de tipo "junta perdida" que garantizara mayor aislamiento de estos compartimentos.

Posterior a esta reestructuración se realizó nuevamente el control de la calidad del aire de las habitaciones con presión positiva, observando que el nivel de criticidad fue bajo (véase tabla 3 ); razón por la que no se tomaron cultivos de las superficies luego de la intervención. La Unidad de Oncología Pediátrica fue abierta nuevamente seis meses después sin presentarse casos nuevos hasta la fecha de infecciones por Fusarium spp.

\begin{tabular}{|c|c|c|c|c|c|c|}
\hline \multirow{2}{*}{$\begin{array}{l}\text { Habi- } \\
\text { tación }\end{array}$} & \multirow[t]{2}{*}{ Lugar } & \multicolumn{2}{|c|}{ Antes de la intervención } & \multicolumn{3}{|c|}{ Luego de intervención final } \\
\hline & & $\mathrm{UFC} / \mathrm{m}^{3}$ & $\begin{array}{l}\text { Microorganismos } \\
\text { visualizados }\end{array}$ & $\mathrm{UFC} / \mathrm{m}^{3}$ & $\begin{array}{l}\text { Microorganismos } \\
\text { visualizados }\end{array}$ & $\begin{array}{l}\text { Critici- } \\
\text { dad* }\end{array}$ \\
\hline \multirow[t]{3}{*}{ A } & $\begin{array}{l}\text { Centro } \\
\text { habitación }\end{array}$ & \multirow{3}{*}{\multicolumn{2}{|c|}{$\begin{array}{l}\text { No se tomaron cultivos de } \\
\text { aire en esta habitación } \\
2 \\
3\end{array}$}} & 0 & Ninguno & \multirow[t]{3}{*}{ Baja } \\
\hline & Esclusa & & & $\begin{array}{l}1 \text { colonia de hongo } \\
\text { filamentoso am- } \\
\text { biental } \\
1 \text { colonia de leva- } \\
\text { dura }\end{array}$ & Baja & \\
\hline & Baño & & & $\begin{array}{l}\text { Hongos filamento- } \\
\text { sos ambiental }\end{array}$ & Baja & \\
\hline \multirow[t]{3}{*}{ B } & $\begin{array}{l}\text { Centro } \\
\text { habitación }\end{array}$ & \multirow{3}{*}{\multicolumn{2}{|c|}{$\begin{array}{l}\text { No se tomaron cultivos de } \\
\text { aire en esta habitación } \\
10 \\
2\end{array}$}} & 1 & $\begin{array}{l}\text { Hongo filamentoso } \\
\text { ambientales }\end{array}$ & \multirow[t]{3}{*}{ Baja } \\
\hline & Esclusa & & & $\begin{array}{l}\text { Hongos filamento- } \\
\text { sos ambientales }\end{array}$ & Baja & \\
\hline & Baño & & & $\begin{array}{l}\text { Hongos filamento- } \\
\text { sos ambientales }\end{array}$ & Baja & \\
\hline \multirow[t]{3}{*}{ C } & $\begin{array}{l}\text { Centro } \\
\text { habitación }\end{array}$ & \multirow{3}{*}{\multicolumn{2}{|c|}{$\begin{array}{l}\text { No se tomaron cultivos de } \\
\text { aire en esta habitación } \\
3 \\
1\end{array}$}} & 0 & Ninguno & \multirow[t]{3}{*}{ Baja } \\
\hline & Esclusa & & & $\begin{array}{l}\text { Hongos filamento- } \\
\text { sos ambientales }\end{array}$ & Baja & \\
\hline & Baño & & & $\begin{array}{l}\text { Hongo filamentoso } \\
\text { ambiental }\end{array}$ & Baja & \\
\hline \multirow[t]{3}{*}{ D } & $\begin{array}{l}\text { Centro } \\
\text { habitación }\end{array}$ & \multirow{3}{*}{\multicolumn{2}{|c|}{$\begin{array}{l}\text { No se tomaron cultivos de } \\
\text { aire en esta habitación } \\
5 \\
0\end{array}$}} & 0 & Ninguno & \multirow[t]{3}{*}{ Baja } \\
\hline & Esclusa & & & $\begin{array}{l}\text { Hongo filamentoso } \\
\text { ambiental }\end{array}$ & Baja & \\
\hline & Baño & & & Ninguno & Baja & \\
\hline
\end{tabular}




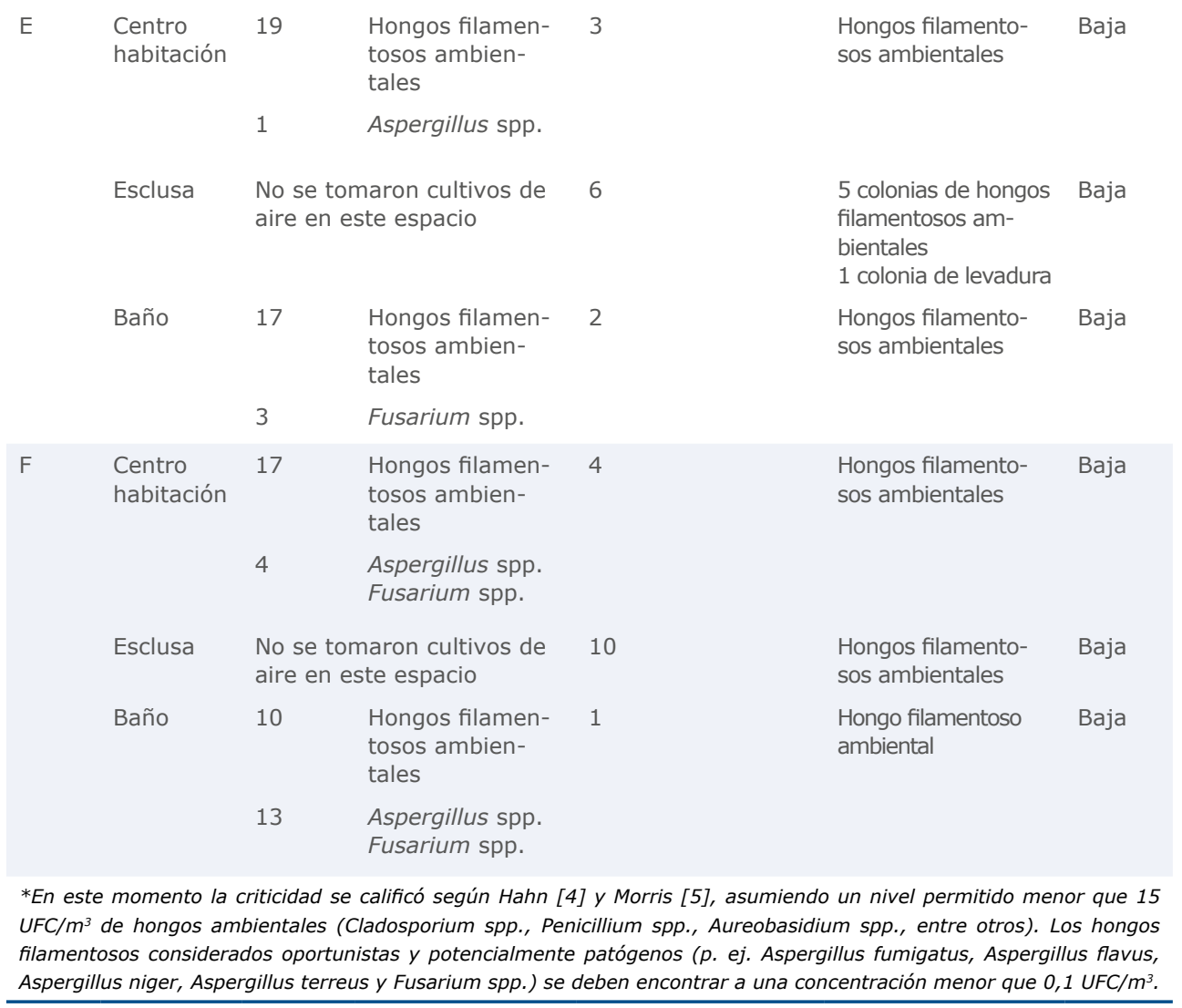

\section{Discusión}

En los servicios médicos es importante contar con una adecuada vigilancia epidemiológica que permita detectar el aumento no esperado de casos de infecciones para así analizar y tomar medidas de control. En este artículo se presenta el hallazgo de un incremento en los aislamientos microbiológicos de Fusarium spp. en la sangre de pacientes hospitalizados en el servicio de Oncología Infantil en un corto periodo. Los niños afectados tenían factores de riesgo para la infección por este hongo, pero la mayoría no presentaron manifestaciones clínicas de infección sistémica por el mismo, lo que hizo sospechar que se trataba posiblemente de casos de pseudofungemias asociadas a algún factor externo o ambiental.

La consideración de pseudofungemias se evidencia, además, en que la mayoría de los pacientes (tres de cinco) tuvieron hemocultivos de control negativos sin haber recibido tratamiento antifúngico dirigido con actividad contra Fusarium spp. (véase tablas 1 y 2). Llama la atención que siendo pacientes profundamente neutropénicos no hayan presentado una infección sintomática por Fusarium spp., lo que se podría deber a un inóculo bajo que pudo haber sido suficiente para causar la colonización de 
los dispositivos más no la infección sistémica; aunque de igual forma se alertó sobre el riesgo al que estaban expuestos los pacientes.

En este estudio de brote hospitalario se consideró que la fuente más probable de contaminación fueron las conidias de Fusarium spp. presentes en el aire de la habitación, que al asentarse contaminan las superficies inanimadas del área y pueden colonizar los dispositivos invasivos del paciente. De esta forma, se podría considerar que los catéteres venosos centrales de los pacientes del estudio fueron colonizados por este hongo, lo cual se evidenció por medio del resultado positivo de los hemocultivos tomados a través de estos dispositivos.

En uno de los casos se puede concluir que el origen de la fungemia fueron los nódulos subcutáneos en el paciente, ya que no tenía dispositivos invasivos, por lo cual recibió tratamiento dirigido contra Fusarium spp.; sin embargo, al haberse presentado en el mismo periodo se consideró parte de este brote. De los cuatro pacientes con dispositivos se puede considerar que solo uno, el cual estaba en tratamiento con voriconazol para una aspergilosis pulmonar previamente diagnosticada, presentó una fungemia asociada a catéter, que fue interpretada por los médicos tratantes como una colonización del dispositivo sin relevancia clínica.

Diferentes autores han publicado estadísticas de fusariosis en pacientes con malignidades hematológicas, describiendo sus desenlaces, presentación, definiendo inclusive factores de pronóstico y algunos relacionando la fuente de infección en el mismo paciente como las lesiones en piel $[6,7]$. Sin embargo, a diferencia de los brotes por Aspergillus spp., que en la literatura están ampliamente relacionados con el aire y los ductos del aire acondicionado contaminados [8], en el caso de la fusariosis son pocos los estudios que evidencian su relación con la seguridad ambiental. Por ejemplo, Litinov y colaboradores (2015) [9] publicaron el análisis de un brote causado por Fusarium spp. en una unidad oncológica de niños, el cual fue aislado del aire y agua de la habitación de los pacientes y que se controló un año después con el mejoramiento de la calidad del aire y la aplicación de filtros en todos los grifos de las habitaciones.

El mantenimiento de la calidad del aire en ambientes controlados es fundamental para reducir la incidencia de micosis invasivas. El nivel adecuado de bioseguridad ambiental para los hongos oportunistas hace referencia a niveles aceptables de contaminación con esporas fúngicas que hace improbable que los enfermos susceptibles adquieran un proceso infeccioso facilitado por el aire. La medición de la contaminación fúngica se debe considerar como un complemento al correcto mantenimiento higiénico del sistema de ventilación y control rutinario de su funcionamiento [10].

Aunque está claro que hay unas situaciones en las que se hace necesario el control microbiológico del aire para verificar la bioseguridad ambiental, por ejemplo, cuando hay detección de anomalías o averías en el funcionamiento del sistema de ventilación, localización de humedades en techos o paredes y aparición de casos de infec- 
ción adquirida en el hospital por hongos oportunistas, antes de ocupar un área recién reestructurada o durante la realización de obras cercanas, aún existe controversia en que el control microbiológico rutinario (periodicidad predefinida) se asocie con una menor tasa de infecciones asociadas al cuidado de la salud [10].

Otros aspectos controversiales en los controles microbiológicos del aire en ambientes controlados son: a) la interpretación de los recuentos fúngicos ante la ausencia de evidencia suficiente en relación con el riesgo de infección y de una norma establecida y b) la falta de consenso para establecer las especies que se puedan considerar como potencialmente patógenas $[10,11]$.

Países como Estados Unidos [12], Reino Unido y Francia [13] han dado directrices oficiales sobre estos controles; sin embargo, es España el que, a través de la Asociación Española de Normalización y Certificación (AENOR), publica normas que ha ido revisando en el tiempo, cuya última versión es la del año 2012 en la Norma UNE 171340. Esta norma define como el nivel óptimo menor que $1 \mathrm{UFC} / \mathrm{m}^{3}$ de hongos filamentosos como Aspergillus spp., Mucor spp., Scedosporium spp., Fusarium spp., Rhizopus spp., entre otros, para habitaciones de ambiente controlado [14-16].

Como producto del análisis del brote del presente manuscrito se tomó la decisión de no hospitalizar los pacientes neutropénicos en habitaciones que no cumplan con las condiciones de ambiente controlado. Además, el presente artículo plantea la dificultad de realizar un seguimiento exhaustivo a este tipo de servicios con ambiente controlado ante la ausencia de la reglamentación propia y la no contundencia de la evidencia disponible.

Algunas limitaciones de este estudio fue la no realización de análisis moleculares para determinar la clonalidad de las cepas, debido a la no disponibilidad de recursos. Al inicio del brote se tomaron muestras ambientales (de aire y superficies) en dos habitaciones de ambiente controlado y en una habitación sin presión positiva, lo cual permitió evidenciar un problema que era común a todas las habitaciones del servicio. Luego de la segunda intervención planteada se tomaron cultivos de aire ambiental a la totalidad de las habitaciones con ambiente controlado para corroborar el control de la situación. Lo anterior se plantea como otra limitación del estudio, ya que no permite hacer una comparación de un antes y después de la intervención en cada una de las habitaciones.

\section{Conclusiones}

El análisis de este pseudobrote hospitalario pone de manifiesto la importancia del control ambiental de las habitaciones que albergan pacientes inmunocomprometidos y al mismo tiempo las dificultades en el seguimiento de su control debido a la poca evidencia disponible. 


\section{Bibliografía}

1. Garcia-Ruiz JC, Amutio E, Ponton J. [Invasive fungal infection in immunocompromised patients]. Rev Iberoam Micol 2004; 21: 55-62.

2. Hospenthal DR. Uncommon Fungi and Prototheca. En: Mandell Gl, Bennett JE, Dolin R, eds. Mandell, Douglas, and Bennett's Principles and Practice of Infectious Diseases. Vol. 2 (ed 7a). Filadelfia, Estados Unidos: Churchill Livingstone, Elsevier; 2010: 3365-3376.

3. Verweij PE, Brandt ME. Aspergillus, Fusarium, and Other Opportunistic Moniliaceous Fungi. En: Murray PR, Baron EJ, Jorgensen JH, Landry ML, Pfaller MA, eds. Manual of Clinical Microbiology. Vol. 2 (ed 9a). Washington D.C., Estados Unidos: ASM Press; 2007: 1802-1838.

4. Hahn T, Cummings KM, Michalek AM, Lipman BJ, Segal BH, McCarthy PL, Jr. Efficacy of high-efficiency particulate air filtration in preventing aspergillosis in immunocompromised patients with hematologic malignancies. Infect Control Hosp Epidemiol 2002; 23: 525-531.

5. Morris G, Kokki MH, Anderson K, Richardson MD. Sampling of Aspergillus spores in air. J Hosp Infect 2000; 44: 81-92.

6. Muhammed $\mathbf{M}$, Anagnostou $\mathbf{T}$, Desalermos A, Kourkoumpetis TK, Carneiro HA, Glavis-Bloom J, et al. Fusarium infection: report of 26 cases and review of 97 cases from the literature. Medicine (Baltimore) 2013; 92: 305-316.

7. Campo M, Lewis RE, Kontoyiannis DP. Invasive fusariosis in patients with hematologic malignancies at a cancer center: 1998-2009. J Infect 2010; 60: 331-337.

8. Lutz BD, Jin J, Rinaldi MG, Wickes BL, Huycke MM. Outbreak of invasive Aspergillus infection in surgical patients, associated with a contaminated air-handling system. Clin Infect Dis 2003; 37: 786-793.

9. Litvinov N, da Silva MT, van der Heijden IM, Graca MG, Marques de Oliveira L, Fu L, et al. An outbreak of invasive fusariosis in a children's cancer hospital. Clin Microbiol Infect 2015; 21: 268 e261-267.

10. Robles García M, Dierssen Sotos T, Llorca Díaz FJ, Rodríguez Cundín P, Roiz Mesones MP. Prevención de la infección nosoco- mial de origen fúngico: verificación de la bioseguridad ambiental en quirófanos. Rev Clin Esp 2005; 205: 601-606.

11. Reino de España, Instituto Nacional de la Salud (INSALUD), Grupo de trabajo de la Sociedad Española de Medicina Preventiva - Salud Pública e Higiene. Recomendaciones para la Verificación de la Bioseguridad Ambiental (BSA) respecto a Hongos Oportunistas. Madrid, España. 2000. Disponible: http://www.sempsph.com/images/stories/ recursos/pdf/protocolos/2012/108_Bioseguridad_Ambiental_frente_a_Hongos.pdf. Consultado: jun 2016.

12. Centers for Disease Control and Prevention (CDC). Guidelines for Environmental Infection Control in Health-Care Facilities: Recommendations of CDC and the Healthcare Infection Control Practices Advisory Committee (HICPAC). MMWR 2003; 52 (No. RR-10): 1-48.

13. República Francesa, Ministerio de SaIud, Dirección General de Salud, Dirección de Hospitalización y Organización Sanitaria, Comisión Técnica Nacional de Infecciones Nosocomiales. Surveillance microbiologique de l'environnement dans les établissements de santé: Air, eaux et surfaces. Francia. 2002. Disponible: http://nosobase.chu-lyon.fr/recommandations/Ministere_Sante/2002_environnement_ministere. pdf. Consultado: jun 2016

14. Reino de España, Asociación Española de Normalización y Certificación (AENOR). Norma UNE 171340: Validación y cualificación de salas de ambiente controlado en hospitales. Madrid, España: AENOR. 2012.

15. Reino de España, Sociedad Andaluza de Medicina Preventiva y Salud Pública. Recomendaciones para la monitorización de la calidad microbiologica del aire (bioseguridad ambiental) en zonas hospitalarias de riesgo. 2014. Disponible: http://www.sociedadandaluzapreventiva.com/wp-content/uploads/ Borrador-protocolo-bioseguridad-SAMPSP. pdf. Consultado: jun 2016.

16. Pemán J, Salavert M. Epidemiología y prevención de las infecciones nosocomiales causadas por especies de hongos filamentosos y levaduras. Enferm Infecc Microbiol Clin 2013; 31: 328-341. 


\section{Creemos en los sueños de todos los colombianos que llevan en la sangre confianza}

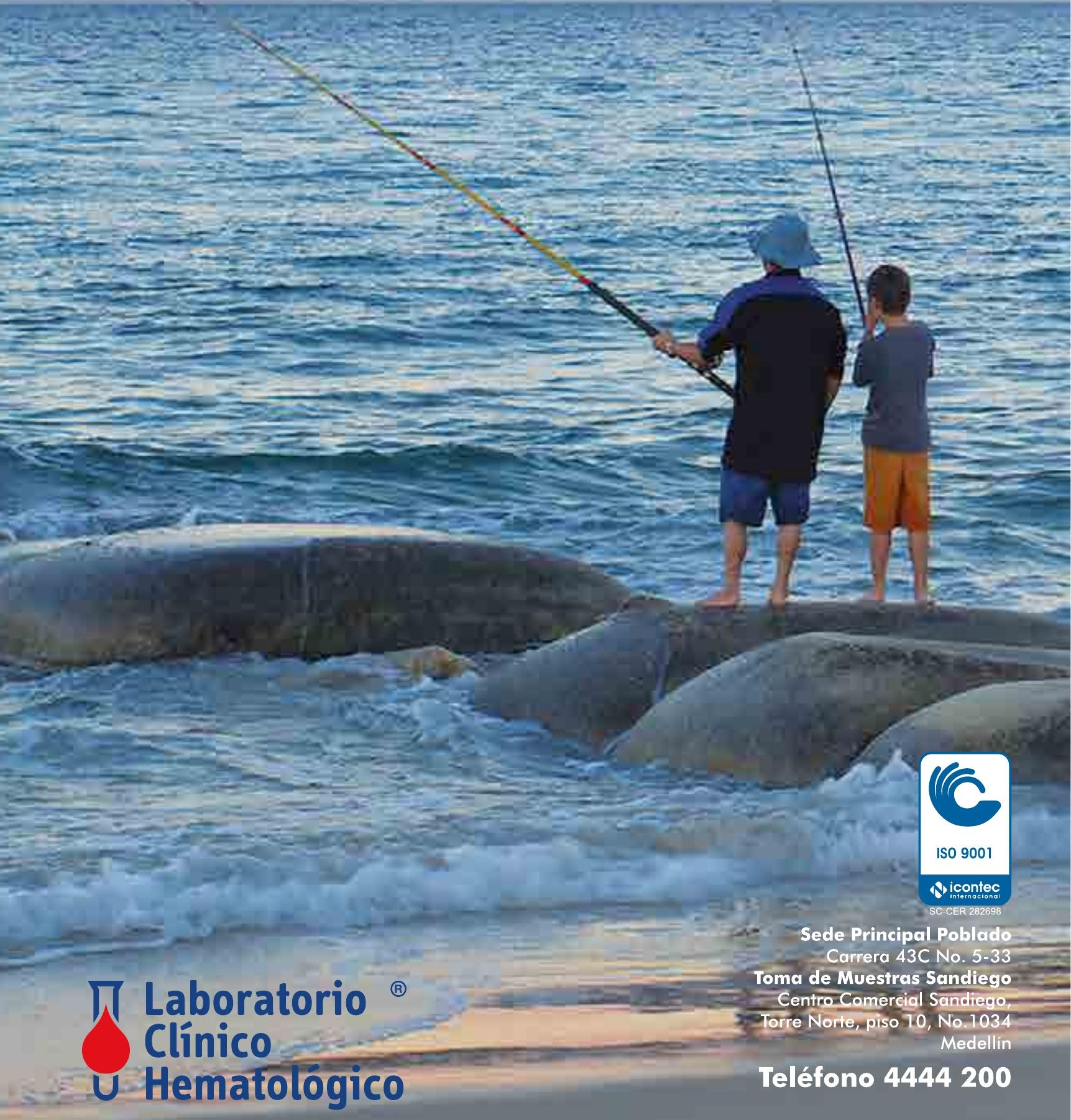

